\title{
Avaliação da cultura de segurança do paciente no centro cirúrgico: um estudo transversal
}

RESUMO | Objetivo: identificar os pontos fortes e frágeis na Cultura de Segurança do Paciente (CSP) em um centro cirúrgico. Método: Trata-se de um estudo transversal realizado Hospital público terciário de referência na região do Cariri, Nordeste do Brasil. Participaram da pesquisa profissionais de saúde da área de enfermagem, que atenderam aos critérios de inclusão: ser profissional da área de enfermagem e trabalhar no mínimo 20 horas semanais. Foram exclusos os profissionais associados e/ou vinculados a cooperativa e os que ocupavam funções administrativas. Resultados: Dentre dimensões da CSP destacaram-se: aprendizagem organizacional continuada 93,8\% e expectativas/ações da direção 85,6\%. Enquanto as dimensões resposta não punitivas para erros $(26,6 \%)$ e abertura para comunicações e mudança de turno/transições $(65,8 \%)$, obtiveram os menores resultados. Conclusão: A liderança pode ser de fundamental importância para construção da CSP forte, a partir do aperfeiçoamento da comunicação entre a equipe e consequentemente remoção da cultura de culpa individual por erro.

Palavras-chaves: Segurança do Paciente; Centro Cirúrgico; Eventos adversos; Cirurgia.

\begin{abstract}
Objective: to identify the strengths and weaknesses in the Patient Safety Culture (PSC) in a surgical center. Method: This is a cross-sectional study conducted in a tertiary public hospital in the Cariri region, Northeast Brazil. Participated in the research health professionals in the nursing area, who met the inclusion criteria: being a nursing professional and working at least 20 hours per week. Professionals associated and/or linked to the cooperative and those who occupied administrative functions were excluded. Results: Among CSP dimensions the following stood out: continued organizational learning $93.8 \%$ and management expectations/actions $85.6 \%$. While the dimensions non-punitive response to mistakes (26.6\%) and openness to communication and change of shift/transitions (65.8\%), obtained the lowest results. Conclusion: Leadership can be of fundamental importance for building a strong CSP, by improving communication among the team and consequently removing the culture of individual blame for errors.
\end{abstract}

Keywords: Patient Safety; Surgical Center; Adverse Events; Surgery.

RESUMEN | Objetivo: Identificar los puntos fuertes y débiles de la Cultura de Seguridad del Paciente (CSP) en un centro quirúrgico. Método: Se trata de un estudio transversal realizado en un hospital público terciario de referencia en la región de Cariri, Nordeste de Brasil. Participaron en la investigación profesionales sanitarios del área de enfermería, que cumplían los criterios de inclusión: ser profesional de enfermería y trabajar al menos 20 horas semanales. Se excluyeron los profesionales asociados y/o vinculados a la cooperativa y los que ocupaban funciones administrativas. Resultados: Entre las dimensiones del CSP destacan: el aprendizaje organizativo continuado $93,8 \%$ y las expectativas/acciones de la dirección $85,6 \%$. Mientras que las dimensiones respuesta no punitiva a los errores $(26,6 \%)$ y apertura a las comunicaciones y a los turnos/transiciones $(65,8 \%)$, obtuvieron los resultados más bajos. Conclusión: El liderazgo puede ser de fundamental importancia para la construcción de un CSP fuerte, a partir de la apertura de la comunicación entre el equipo y la consecuente remoción de la cultura de la culpa individual por el error.

Palabras claves: Seguridad del paciente; Centro quirúrgico; Eventos adversos; Cirugía.

\section{Letícia Lima Cruz}

Enfermeira graduada pela Faculdade de Medicina Estacio de Juazeiro do Norte - FMJ(CE). ORCID: 0000-0003-3306-9138

\section{Raimundo Monteiro da Silva Neto}

Graduação em enfermagem em andamento pelo Centro Universitário de Juazeiro do Norte (UNIJUAZEIRO), Juazeiro do Norte (CE), Brasil.

ORCID: 0000-0001-9949-1740

\section{José Lucas Souza Ramos}

Enfermeiro. Mestre em Ciências (Obstetrícia e Ginecologia) pela Faculdade de Medicina da Universidade de São Paulo (USP) (SP) Professor do Curso de Enfermagem e da Pós-graduação Lato Sensu da Escola Superior de Ciências da Santa Casa de Misericórdia de Vitória e Tutor da Residência
Multiprofissional em Cuidados Paliativos do ICEPi/SESA, Governo do Estado (ES). ORCID: 0000-0002-6985-9716

\section{Nayanna Karla Alves Feitosa}

Enfermeira pelo Centro Universitário de Juazeiro do Norte (CE).

ORCID: 0000-0003-0915-771X

\section{Italla Maria Pinheiro Bezerra}

Enfermeira. Pós-doutorado pela Universidade de São Paulo EACH-USP; Doutorado em Ciências (área de concentração: saúde Coletiva). Setor de Pós-graduação, Pesquisa e Inovação, Centro Universitário Saúde $A B C$, FMABC, Santo André, SP, Brazil, Programa de Pós-graduação em Políticas Públicas e Desenvolvimento Local, Escola Superior de Ciências da Santa Casa de Misericórdia de Vitória, EMESCAM, Vitória, ES, Brazil, Laboratório de Escrita Científica,
Escola Superior de Ciências da Santa Casa de Misericórdia de Vitória, EMESCAM, Vitória, (ES), Brasi.

ORCID: 0000-0002-8604-587X

\section{Cíntia de Lima Garcia}

Enfermeira. Doutorado em Ciências com ênfase em Saúde Coletiva (2019) Centro Universitário Saúde $A B C$, FMABC, Santo André (SP), Brasil. Docente dos cursos de Medicina e Enfermagem da Faculdade de Medicina Estácio de Juazeiro do Norte (ESTACIO FMJ). Coordenadora do Laboratório de Habilidades e Simulação Realística (LHS) da ESTACIO FMJ. Coordenadora de período do curso de Medicina da ESTACIO FM Juazeiro do Norte (CE).

ORCID: 0000-0003-2673-7003

Recebido em: 12/05/2021

Aprovado em: 18/05/2021 
$\Lambda$ cultura de segurança do paciente (CSP) é resultado de um conjunto de valores e competências que instituem um padrão de comportamento ${ }^{1}$. Uma instituição com cultura de segurança positiva, sinaliza a oferta de uma assistência com maior qualidade aos seus usuários. Entretanto, um grande desafio ainda é a frequência e multifatoriedade de eventos adversos ${ }^{2}$.

O centro cirúrgico é um dos setores com maior incidência de eventos adversos $^{3}$, durante todo o perioperatório o paciente é exposto a vários riscos como: negligência no uso do checklist de cirurgia segura e déficit na comunicação entre os profissionai ${ }^{s 4}$. De acordo com a OMS, anualmente no mínimo 7 milhões de pacientes são prejudicados por complicações cirúrgicas, destes, aproximadamente 1 milhão evoluem a óbito.

A gerência de uma instituição é responsável por planejar e implementar estratégias voltadas para uma boa CSP. Contudo, é necessário que cada membro da equipe possua corresponsabilidade para promover segurança ${ }^{5}$. Estima-se que no ano de 2030 mais de 23 milhões de pessoas poderão necessitar de procedimentos cirúrgicos. Com este aumento, podemos inferir que o centro cirúrgico pode precisar de ainda mais particularidades para garantir a segurança do paciente $(\mathrm{SP})^{1,3,6}$.

Sabe-se que a equipe de enfermagem é a categoria com maior número de profissionais presentes no setor, por isso, precisa estar engajada nas ações educacionais e assistenciais seguras ${ }^{1}$. Diante disso, questionou-se: a cultura de segurança do paciente do centro cirúrgico encontra-se adequada para prevenção de eventos adversos? A partir de uma avaliação da cultura de segurança no setor, pode-se desenvolver uma visão capaz de identificar as fragilidades na segurança ${ }^{1}$ e traçar estratégias para promover uma assistência segura e diminuir danos ${ }^{7}$.

O presente estudou objetivou identi-

ficar os pontos fortes e frágeis na CSP em um centro cirúrgico.

\section{METODO}

A cultura de segurança do paciente (CSP) é resultado de um conjunto de valores e competências que instituem um padrão de comportamento. Uma instituição com cultura de segurança positiva, sinaliza a oferta de uma assistência com maior qualidade aos seus usuários. Entretanto, um grande desafio ainda é a frequência e multifatoriedade de eventos adversos.
Trata-se de uma pesquisa transversal, descritiva com abordagem quantitativa, onde todas análises foram feitas em um curto período de tempo e posteriormente foram descritas as características da CSP identificadas ${ }^{8}$. O estudo foi realizado em um Hospital público terciário de referência na região do Cariri, Nordeste do Brasil. Participaram da pesquisa profissionais de saúde da área de enfermagem: enfermeiros e técnicos de enfermagem, que atenderam aos critérios de inclusão: ser profissional da área de enfermagem e trabalhar no mínimo 20 horas semanais. Foram exclusos os profissionais associados e/ou vinculados a cooperativa e os que ocupavam funções administrativas.

A coleta de dados ocorreu entre os meses de fevereiro e abril de 2020, de maneira presencial e online: portando um tablet, os profissionais foram abordados de acordo com sua disponibilidade temporal, no qual estava disposto o questionário. No método online, o participante cadastrado recebeu o questionário auto administrado no seu e-mail para respondê-lo sem necessidade de um entrevistador. $\mathrm{O}$ instrumento utilizado nessa referida pesquisa, foi o questionário Hospital Survey on Patient Safety Culture (HSOPSC), em uma versão adaptada, validada, traduzida para a realidade brasileira ${ }^{9}$. Composto por 42 questões que proporcionaram avaliar a CSP a partir de 12 Dimensões $(\mathrm{D})^{1,10}$.

A pesquisa envolveu riscos mínimos e respeitou todos os aspectos éticos e legais dispostos na resolução 466/12 que orienta as pesquisas científicas e resguardam os participantes ${ }^{11}$, CAAE da pesquisa: 17148819.10000.5684. Após a aprovação ética foram solicitados: nomes completos, data de admissão, e-mail dos profissionais e áreas de atuação/setor. Todos os profissionais cadastrados da assistência de enfermagem fo- 
Tabela 1 - Caracterização da amostra Juazeiro do Norte- CE, Brasil, 2020

\begin{tabular}{lccc} 
& Sexo & $\mathbf{n}$ & Porcentagem \\
Masculino & 10 & $20,8 \%$ \\
\hline Feminino & 38 & $79,2 \%$ \\
\hline Profissão & & \\
\hline Enfermeiro & 11 & $22,9 \%$ \\
\hline Técnico de Enfermagem & 37 & $77,1 \%$
\end{tabular}

Experiência na área

\begin{tabular}{lcc|}
\hline Menos de 1 ano & 5 & $10,6 \%$ \\
\hline De 2 a 5 anos & 14 & $29,8 \%$ \\
\hline De 6 a 10 anos & 17 & $36,2 \%$ \\
\hline De 11 a 15 anos & 2 & $4,3 \%$ \\
\hline De 16 a 20 anos & 1 & $2,1 \%$ \\
\hline 21 anos ou mais & 1 & $2,1 \%$ \\
\hline Tempo de trabalho no hospital & & \\
\hline Menos de 1 ano & 3 & $6,4 \%$ \\
\hline De 2 a 5 anos & 15 & $31,9 \%$ \\
\hline De 6 a 10 anos & 20 & $42,6 \%$ \\
\hline Carga horária semanal & & \\
\hline Até 20 horas & 13 & $27,7 \%$ \\
\hline De 21 a 39 horas & 8 & $17 \%$ \\
\hline 40 horas ou mais & 26 & $55,3 \%$ \\
\hline Contato com o paciente & & \\
\hline SIM, eu costumo ter contato com os pacientes & 45 & $97,8 \%$ \\
\hline NÃO, eu NÃO costumo ter contato com os pacientes & 1 & $2,2 \%$ \\
\hline Total: & 48 & $100 \%$ \\
\hline Fonte: Dados da pesquisa Juazeiro do Norte-CE, Brasil, 2020 & & \\
\hline
\end{tabular}

Tabela 2 - Porcentagem de respostas por dimensão Juazeiro do Norte- CE, Brasil, 2020

\begin{tabular}{lc}
\multicolumn{1}{c}{ Dimensões } & $\%$ \\
\hline 1 - Frequência de eventos notificados & $77,1 \%$ \\
\hline 2 - Percepção de segurança & $74,5 \%$ \\
\hline $\begin{array}{l}3 \text { - Expectativas e ações da direção/supervisão da unidade/serviço que favorecem a } \\
\text { segurança }\end{array}$ & $85,6 \%$ \\
\hline 4 - Aprendizagem organizacional/melhoria continuada & $93,8 \%$ \\
\hline 5 - Trabalho em equipe na unidade/serviço & $83,2 \%$ \\
\hline 6 - Abertura para comunicações & $60,2 \%$ \\
\hline 7 - Feed-back e comunicação sobre erros & $77,0 \%$ \\
\hline 8 - Resposta não punitivas para erros & $26,6 \%$ \\
\hline 9 - Dimensionamento de pessoal & $66,5 \%$ \\
\hline 10 - Apoio da gerência do hospital para a segurança do paciente & $84,0 \%$ \\
\hline 11 - Trabalho em equipe entre unidades & $69,3 \%$
\end{tabular}

ram classificados por número de ordem, de acordo com os critérios de inclusão para participar do estudo.

Para análise de dados foi utilizado o programa computacional a metodologia XP (Extreme Programming. Esse programa computacional viabiliza exportar os dados para análise mais detalhada em outros softwares, como Excel, Statistical Package for the Social Sciences (SPSS) e Google Drive 10. Proporciona também a construção de tabelas e gráficos de indicadores para o percentual de respostas positivas $(>75 \%)$, neutras $(>50 \% \mathrm{e}<$ $75 \%)$ e negativas $(<50 \%)$, em comparação aos itens individuais, às dimensões da cultura de segurança e do questionário geral ${ }^{10,12}$.

\section{RESULTADOS}

A amostra foi composta por 48 profissionais de saúde que trabalhavam no Centro Cirúrgico com carga horária de trabalho semanal de 40 horas ou mais. Desse total de participantes, 97,8\% referem ter contato direto com o paciente (Tabela 1).

Dentre as 12 dimensões da Cultura de Segurança do Paciente avaliadas pelo HSPOSC, as que obtiveram meIhores resultados foram: aprendizagem organizacional/melhoria continuada $(93,8 \%)$; expectativas e ações da direção/supervisão da unidade/serviço que favorecem a segurança $(85,6 \%)$; e, apoio da gerência do hospital para a segurança do paciente (84\%).

Enquanto que, as dimensões "resposta não punitivas para erros", "abertura para comunicações" e "problemas em mudanças de turno e transições entre unidades/serviços", obtiveram os resultados mais baixos, conforme indicado na Tabela 2.

Identificou-se que a dimensão "Resposta não punitiva para erros", foi a única que apresentou percentual negativo $(26,6 \%)$, sendo caracterizada como uma área frágil para CSP. 
12 - Mudanças de turno e transições entre unidades/serviços

Fonte: Dados da pesquisa Juazeiro do Norte-CE, Brasil, 2020

\begin{tabular}{|c|c|c|c|}
\hline Dimensões & + & * & - \\
\hline \multicolumn{4}{|l|}{ Resposta não punitiva para erros } \\
\hline $\begin{array}{l}\text { 1.0s profissionais consideram que seus erros podem ser usados } \\
\text { contra eles }\end{array}$ & $24,4 \%$ & $8,9 \%$ & $66,7 \%$ \\
\hline $\begin{array}{l}\text { 2.Quando um evento é notificado, parece que o foco recai sobre a } \\
\text { pessoa e não sobre o problema }\end{array}$ & $41,7 \%$ & $22,9 \%$ & $35,4 \%$ \\
\hline $\begin{array}{l}\text { 3.Os profissionais temem que seus erros sejam registrados em suas } \\
\text { fichas funcionais }\end{array}$ & $13 \%$ & $17,4 \%$ & $69,6 \%$ \\
\hline
\end{tabular}

+=Positivo, -=Negativo *= respostas neutras Fonte: Dados da pesquisa Juazeiro do Norte-CE, Brasil, 2020

Tabela 4- Avaliação dos itens das dimensões Juazeiro do Norte-CE, Brasil, 2020

$$
\text { Dimensões } \quad+1 * 1-\%
$$

Expectativas e ações da direção/supervisão da unidade/serviço que favorecem a segurança

1. 0 meu supervisor/chefe elogia quando vê um trabalho realizado de acordo com os procedimentos estabelecidos de segurança do paciente

$76,6 / 12,8 / 10,6 \%$

2. 0 meu supervisor/chefe realmente leva em consideração as sugestões dos profissionais para melhoria da segurança do paciente

$85,1 / 10,6 / 4,3 \%$

3.Sempre que a demanda aumenta, meu supervisor/chefe quer que trabaIhemos mais rápido, mesmo que isso signifique "pular etapas"

$83,0 / 8,5 / 8,5 \%$

4. 0 meu supervisor/chefe não dá atenção suficiente aos problemas de
segurança do paciente que acontecem repetidamente

Aprendizagem organizacional/melhoria continuada

5. Estamos adotando medidas para melhorar a segurança do paciente $93,8 / 6,2 \% /-$

6. Quando se identifica um erro na atenção ao paciente, adotamos medidas para preveni-lo

$97,9 /-/-$

7. Após implementarmos mudanças para melhorar a segurança do paciente, avaliamos sua efetividade

$89,6 / 6,2 / 4,2 \%$

Apoio da gerência do hospital para a segurança do paciente

8. A direção do hospital propicia um clima de trabalho que promove a segurança do paciente

$89,6 / 6,2 / 4,2 \%$

9. As ações da direção do hospital demonstram que a segurança do paciente é uma prioridade

$85,4 / 10,4 / 4,2 \%$

10. A direção do hospital só demonstra interesse na segurança do paciente quando ocorre algum evento adverso

$77,1 / 10,4 / 12,5 \%$

Fonte: Dados da pesquisa Juazeiro do Norte-CE, Brasil, 2020

\section{DISCUSSÃO}

Observou-se no estudo que das doze dimensões avaliadas seis obtiveram percentuais acima de $75 \%$, sendo consideradas áreas fortes; cinco apresentaram-se como área em potencial fortalecimento ou neutras com pontuação entre 60$74,5 \%$; e, resposta punitiva para erros obteve resultado negativo apresentando $26,6 \%$, sendo considerada como área frágil para CSP. Pode-se considerar que identificar os pontos fortes e frágeis da CSP possibilita resultados cada vez mais positivos para os pacientes e organizações de saúde ${ }^{13}$.

A comunicação ineficaz tem sido um dos principais fatores relacionados ao cuidado inseguro e a ocorrência de incidentes em instituições hospitalares ${ }^{14}$. De acordo com Pinheiro et al. (2017) e Costa et al. (2018), para estabelecer uma comunicação efetiva, é necessário o envolvimento da gestão em situações cotidianas, como uma maior frequência de reuniões e padronizar a transmissão de informações importantes sobre o paciente no perioperatório ${ }^{15,16}$.

Sobre as mudanças de turno e transições entre unidades, acredita-se que pode não ser considerada como uma área forte pelo fato de muitos profissionais da enfermagem terem baixa remuneração e necessitarem de duplos vínculos. A dupla jornada de trabalho da enfermagem pode favorecer o desgaste físico e psicológico ${ }^{17}$. O estudo de Forte et al. (2019) mostrou que as principais causas está a sobrecarga de trabalho ocasionada por um déficit na quantidade de profissionais ou necessidade de trabalhar em mais de um serviço ${ }^{25}$. A melhoria das condições de trabalho da equipe de enfermagem é considerada fundamental para o fortalecimento da $\mathrm{CSP}^{18}$.

Pesquisas realizadas na Etiópia, ${ }^{19}$ Turquia $^{20}$, Arábia Saudita ${ }^{21}$ e Hungria ${ }^{22}$, onde a dimensão "resposta não punitiva ao erro" foi identificada como área fragilizada. Quando trabalha-se de um modo onde os profissionais não são culpados pelos erros no processo do cuidado e buscam aprender a partir dos erros, acontece então a estruturação de uma cultura justa, pautada na transparência e confiança ${ }^{15}$.

Autores na Hungria observaram que uma "cultura de culpa" prejudica o fortalecimento da $\mathrm{CSP}^{22}$. Um estudo espanhol, em concordância enfatizou que é preciso eliminar a culpa para melhorar a comunicação na equipe ${ }^{23}$. Outro fator é que a resposta punitiva ao erro favore- 
ce a subnotificação, diminui a abertura para comunicação e prejudica o planejamento de processos mais seguros 24 . Sabe-se que se o evento adverso notificado é tratado de maneira meramente punitiva, o profissional terá a tendência em omiti-lo ${ }^{2}$.

Sendo assim, é necessário utilizar ferramentas que aprimorem e promovam um cuidado seguro, tais como a redução das barreiras que dificultam a comunicação entre os membros da equipe cirúrgica e remoção de cultu- ra de culpa para incentivar a notificação de incidentes e eventos adversos ${ }^{7}$. Dessa maneira a prioridade e busca da equipe será trabalhar na prevenção de eventos adversos antes que estes venham a ocorrer e fortalecer a cultura de segurança nos serviços de saúde.

\section{CONCLUSÃO}

O estudo evidenciou que $50 \%$ das dimensões avaliadas pelo estudo foram classificadas como áreas fortes, de modo geral a CSP do setor cirúrgico da pesquisa encontrou-se adequado, e a resposta punitiva foi a área mais crítica, nesta dimensão o setor deve aprimorar-se para melhorar a confiança na equipe e aumentar a qualidade da assistência prestada. A liderança pode atuar no rompimento das barreiras identificadas para construção da CSP forte, a partir do aperfeiçoamento da comunicação entre a equipe e consequentemente remoção da cultura de culpa individual por erro.

\section{Referências}

1. Abreu IM de, Rocha RC, Avelino FVSD, Guimarães DBO, Nogueira LT, Madeira MZ de A. Cultura de segurança do paciente em centro cirúrgico: visão da enfermagem. Rev Gauch Enferm. 2019;40(spe):e20180198. doi:10.1590/19831447.2019.20180198

2. de Lima Garcia C, Bezerra IMP, Ramos JLS, do Valle JETMR, de Oliveira MLB, de Abreu LC. Association between culture of patient safety and burnout in pediatric hospitals. PLoS One. 2019;14(6). doi:10.1371/journal.pone.0218756 3. OMS. CIRURGIAS SEGURAS SALVAM VIDAS MANUAL ALIANÇA MUNDIAL PARA A SEGURANÇA DO PACIENTE SEGUNDO DESAFIO GLOBAL PARA A SEGURANÇA DO PACIENTE.; 2009. www.who.int/patientsafety/en/www.who. int/patientsafety/challenge/safe.surgery/en/. Accessed March 27, 2021.

4. Bohomol E, Tatarli J de A. Utilização de cenários para educação sobre segurança do paciente em centro cirúrgico. Rev SOBECC. 2017:138-144. http:// fi-admin.bvsalud.org/document/view/vu6er. Accessed March 27, 2021.

5. Magalhães FH de L, Pereira IC de A, Luiz RB, Barbosa MH, Ferreira MBG. Clima de segurança do paciente em um hospital de ensino. Rev Gauch Enferm. 2019;40(spe):e20180272. doi:10.1590/1983-1447.2019.20180272

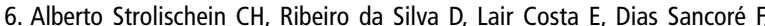
Gustavo Küster Azeredo T, Campos Fontoura F. PREVALÊNCIA DAS PRINCIPAIS COMPLICACÕES PÓS-OPERATÓRIO EM CIRURGIAS CARDÍACAS DE REVASCULARIZAÇÃO DO MIOCÁRDIO EM HOSPITAL FILANTRÓPICO DE CUIABÁ-MT. Vol 5.; 2019. https://revista.ajes.edu.br/index.php/sajes/article/view/305. Accessed April 4, 2021.

7. Batista J, Cruz ED de A, Alpendre FT, Paixão DP da SS da, Gaspari AP, Mauricio AB. Cultura de segurança e comunicação sobre erros cirúrgicos na perspectiva da equipe de saúde. Rev Gauch Enferm. 2019;40(spe):e20180192. doi:10.1590/1983-1447.2019.20180192

8. Hulley SB, Cummings SR, Browner WS, Grady DG, Newman TB. Delineando A Pesquisa Clínica.; 2015. D'siponível em: < https://www.biosanas.com.br/uploads/ outros/artigos_cientificos/143/c4fd11a995cc235510d275cf8298427d.pdf> .

9. Reis CT, Laguardia J, Vasconcelos AGG, Martins M. Confiabilidade e validade da versão brasileira da pesquisa sobre cultura de segurança do paciente em hospitais (HSOPSC): Um estudo piloto. Cad Saude Publica. 2016;32(11). doi:10.1590/0102-311X00115614

10. Andrade LEL de, Melo LOM de, Silva IG da, et al. Adaptação e validação do Hospital Survey on Patient Safety Culture em versão brasileira eletrônica. Epidemiol e Serv saude Rev do Sist Unico Saude do Bras. 2017;26(3):455-468. doi:10.5123/S1679-49742017000300004

11. BRASIL. RESOLUÇÃO No 466, DE 12 DE DEZEMBRO DE 2012; 2013. https://bvsms.saude.gov.br/bvs/saudelegis/cns/2013/res0466_12_12_2012. html. Accessed March 27, 2021.

12. for Healthcare Research A. Hospital Survey on Patient Safety Culture: User's Guide.; 2016. http://www.ahrq.gov. Accessed March 27, 2021.

13. Sanchis DZ, Haddad M do CFL, Girotto E, Silva AMR. Patient safety culture: perception of nursing professionals in high complexity institutions. Rev Bras
Enferm. 2020;73(5):e20190174. doi:10.1590/0034-7167-2019-0174

14. Nogueira JW da S, Rodrigues MCS. EFFECTIVE COMMUNICATION IN TEAMWORK IN HEALTH : A CHALLENGE FOR PATIENT SAFETY *. 2015.Disponível em: < https://www.semanticscholar.org/paper/EFFECTIVE-COMMUNICATION-IN-TEAMWORK-IN-HEALTH-\%3A-A-*-Nogueira-Rodrigues/2443ed6b2dd38ebe252a2881e37487bd4230a338>

15. Pinheiro $M$ da $P$, Junior $O C$ da $S$. Evaluation of the patient safety culture within the hospital organization of a university hospital. Enferm Glob 2017;16(1):339-352. doi:10.6018/eglobal.16.1.238811

16. da Costa DB, Ramos D, Gabriel CS, Bernardes A. Patient safety culture: Evaluation by nursing professionals. Texto e Context Enferm. 2018;27(3) doi:10.1590/0104-070720180002670016

17. Ferri Do Amaral J, Ribeiro JP, Xavier Da Paixão D. QUALIDADE DE VIDA NO TRABALHO DOS PROFISSIONAIS DE ENFERMAGEM EM AMBIENTE HOSPITALAR: UMA REVISÃO INTEGRATIVA Quality of Life at Work of Nursing Professionals in Hospitals: An Integrated Review. Vol 16.; 2015. doi:10.22421/ 15177130-2015V16N1P66

18. Cho $E$, Lee NJ, Kim EY, et al. Nurse staffing level and overtime associated with patient safety, quality of care, and care left undone in hospitals: A cross-sectional study. Int J Nurs Stud. 2016;60:263-271. doi:10.1016/j.ijnurstu.2016.05.009

19. Wami SD, Demssie AF, Wassie MM, Ahmed AN. Patient safety culture and associated factors: A quantitative and qualitative study of healthcare workers' view in Jimma zone Hospitals, Southwest Ethiopia. BMC Health Serv Res. 2016;16(1). doi:10.1186/s12913-016-1757-z

20. Gözlü K, Kaya S. Patient Safety Culture as Perceived by Nurses in a Joint Commission International Accredited Hospital in Turkey and Its Comparison with Agency for Healthcare Research and Quality Data A R T I C L E I N F 0. Vol 4. Mashhad University of Medical Sciences; 2014. doi:10.22038/PSJ.2016.7640 21. Alquwez N, Cruz JP, Almoghairi AM, et al. Nurses' Perceptions of Patient Safety Culture in Three Hospitals in Saudi Arabia. J Nurs Scholarsh. 2018;50(4):422-431. doi:10.1111/jnu.12394

22. Granel N, Manresa-Domínguez JM, Barth A, Papp K, Bernabeu-Tamayo MD. Patient safety culture in Hungarian hospitals. Int J Health Care Qual Assur. 2019:32(2):412-424. doi:10.1108/IJHCQA-02-2018-0048

23. Gutiérrez Ubeda SR. ¿Se necesita un esfuerzo para reemplazar la cultura punitiva por la de seguridad del paciente? Rev Calid Asist. 2016;31(3):173176. doi:10.1016/j.cali.2015.09.007

24. Silva FG da, Junior NJ de O, Oliveira DO de, Nicoletti DR, Comin E. Análise de Eventos Adversos Em Um Centro Cirúrgico Ambulatorial. Vol 20.; 2015. https://revista.sobecc.org.br/sobecc/article/view/91. Accessed March 27, 2021. 25. Forte ECN, de Pires DEP, da Silva Martins MMFP, de Souza Padilha MIC, Schneider DG, de Lima Trindade L. Work process: A basis for understanding nursing errors. Rev da Esc Enferm. 2019;53:1-6. doi:10.1590/S1980$220 \times 2018001803489$ 


\section{INSTITUTO DE SAÚDE E GESTÄO HOSPITALAR - ISGH Plotoforma Brasil}

\section{PARECER CONSUBSTANCIADO DO CEP}

\section{DADOS DO PRONETO DE PESQUISA}

Titulo da Pesquisa: AVALIAÇĂO DA CULTURA DE SEGURANÇA DO PACIENTE NA EQUIPE DE ENFERMAGEM EM UMA INSTITUIÇĀO HOSPITALAR PÚBLICA

Pesquisador: Cintia de Lima Garcia

Área Temática:

Versāo: 1

CAAE: $17148819,1,0000.5684$

Instituiçāo Proponente: INSTITUTO DE SAUDE E GESTÃO HOSPITALAR

Patrocinador Principal: Financiamento Próprio

DADOS DO PARECER

Número do Parecer: 3.488 .076

Apresentaçăo do Projeto:

"Trata-se de uma pesquisa transversal, descritiva com abordagem quantitativa. No Hospital Regional do Cariri (HRC). Participarăo da pesquisa profissionais de saúde da área de enfermagem, tais: Enfermeiros e Técnicos de Enfermagem. Que atendam as seguintes critérios de inclusăo: ser profissional da área de enfermagem está trabalhando no periodo mínimo de doze meses, trabalhar no minimo $20 \mathrm{hs}$ semanais. Os critérios de exclusăo estabelecidos serăo: profissionais associados e/ou vinculados a cooperativa, profissionais que ocupam funçōes administrativas e/ou gerenciais.Sucederá uma visita ao setor de Recursos Humano, autorizado de forma prévia pela gerência de ensino e pesquisa do HRC.O instrumento que será utilizado nessa referida pesquisa, será o questionário o Hospital Survey on Patient Safety Culture (HSOPSC).

\section{Objetivo da Pesquisa:}

Objetivo primário:

- Avaliar a percepçăo dos profissionais de enfermagem sobre a cultura de segurança do paciente em um hospital público.

Objetivos secundários:

- Mensurar os niveis das dimensōes da cultura de segurança do paciente por unidade hospitalar.

- Identificar as áreas com pontos fortes e frágeis dos setores pesquisados através das dimensőes

Endereço: Rua Socorno Gomes, 190

Bair ro: Guajeru

UF: CE Municipio: FORTALEZA

CEP: $60.843-070$

Telefone: $\quad(85) 3195-2767$ Fax: $\quad(85) 3195-2765$

E-mail: cepisghegmal.com 


\section{INSTITUTO DE SAÚDE E GESTÃO HOSPITALAR - ISGH

Conthusça do Parecer: 3488 .076

da cultura de segurança do paciente.

- Averiguar o processo gerencial da cultura de segurança do paciente no serviço, sob a ótica dos profissionais de enfermagem.

Avaliação dos Riscos e Benefícios:

Riscos:

De acordo com a pesquisadora a "pesquisa envolve riscos minimos que podem estar relacionados à algum constrangimento em relaçăo as perguntas relativas a sua prática profissional e/ou a seu locus de assistência. Esse risco será minimizado pela garantia do anonimato, e a garantia que a identidade não será revelada." A aplicaçấo do questionário se dará em ambiente reservado da própria instituição.

\section{Beneficios:}

"Beneficios subsidiar pesquisa futuras e na cooperaçăo na melhoria da qualidade dos serviços prestados da instituiçāo participante. Alem disso, espera-se que os resultados dessa pesquisa polencialmente possam contribuir para o fortalecimento da cultura de segurança do paciente nas instituiçôes e assim, reduçấo da incidencia de eventos adversos."

\section{Comentários e Consideraçőes sobre a Pesquisa:}

Trata-se de um estudo do tipo descritivo e transversal a ser realizado no $\mathrm{HRC}$ sobre a cultura de segurança na equipe de enfermagem. Será aplicado um questionário sobre avaliaçâo da cultura de segurança em ambiente reservado.

\section{Consideraçőes sobre os Termos de apresentaçăo obrigatória:}

Anexados na Plataforma Brasil os documentos obrigatórios para execução da pesquisa: Folha de Rosto; Carta de anuência; Termo de Consentimento Livre Esclarecido; Termo de Ciência da Unidade Hospitalar; Projeto detalhado; Instrumento de coleta de dados; Cronograma; Orçamento.

Recomendaçóes:

- Recomenda-se a comunicação e registro de quaisquer alteraçōes realizadas no protocolo de pesquisa ao Comite de Ética em Pesquisa e Centros Participantes.

- Recomenda-se que ao término da pesquisa, o pesquisador realize a devolutiva dos resultados da pesquisa ao Comitê de Ética em Pesquisa do Instituto de Saúde e Ges tâo Hospitalar por meio do envio do Relatório Final de Pesquisa na aba Notificaçōes da Plataforma Brasil e para a Instituiçăo participante.

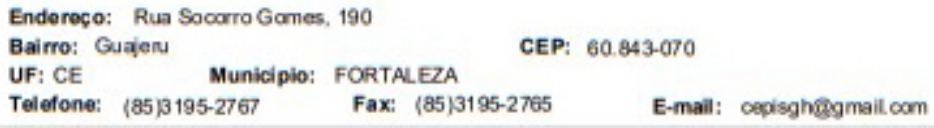




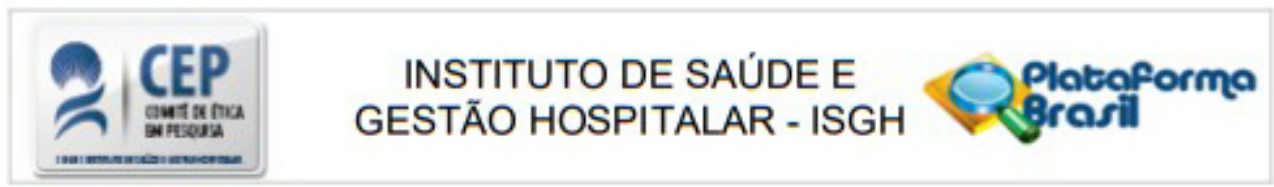

Contrusaça do Parecer: 3488 . 076

Conclusões ou Pendências e Lista de Inadequações:

O trabalho obedece a RDC 466/12, estando apto para ser iniciado.

Consideraçōes Finais a critério do CEP:

O colegiado acata o parecer da relatoria quanto à aprovaçăo do projeto de pesquisa, visto atender a apresentaçāo dos documentos obrigabrios e seguir os preceitos éticos. A pesquisa deve ser desenvolvida mediante delineamento do protocolo aprovado, informando efeitos adversos ou fatos relevantes que alterem o fluxo das normas da pesquisa. Emendas ou modificaçōes ao protocolo devem ser enviadas ao CEP para apreciaçăo ética. Ao término da pesquisa, enviar relatório final para a Instituiçăo participante e CEP/ISGH.

Este parecer foi elaborado baseado nos documentos abaixo relacionados:

\begin{tabular}{|c|c|c|c|c|}
\hline Tipo Documento & Arquivo & Postagem & Autor & Situaçāo \\
\hline $\begin{array}{l}\text { Informaçб̄es Básicas } \\
\text { do Projeto }\end{array}$ & $\begin{array}{l}\text { PB_INFORMAÇOES_BASICAS_DO_P } \\
\text { ROJETO 1363723.pdf }\end{array}$ & $\begin{array}{c}03 / 07 / 2019 \\
17: 24: 42 \\
\end{array}$ & & Aceito \\
\hline Cronograma & Cronograma,docx & $\begin{array}{c}03 / 07 / 2019 \\
17: 24: 09 \\
\end{array}$ & Cintia de Lima Garcia & Aceilo \\
\hline Folha de Rosto & FolhaAssinada.docx & $\begin{array}{c}03 / 07 / 2019 \\
17: 23: 53 \\
\end{array}$ & Cintia de Lima Garcia & Aceito \\
\hline $\begin{array}{l}\text { Projeto Detalhado / } \\
\text { Brochura } \\
\text { Investigador }\end{array}$ & ProjetoCompleb,docx & $\begin{array}{c}03 / 07 / 2019 \\
11: 51: 32\end{array}$ & Cintia de Lima Garcia & Aceito \\
\hline Outros & DeclaracaoCienciaPesquisador,docx & $\begin{array}{c}23 / 05 / 2019 \\
16: 14: 42\end{array}$ & Cintia de Lima Garcia & Aceito \\
\hline Outros & TermodeCiencia,docx & $\begin{array}{c}23 / 05 / 2019 \\
16: 13: 43 \\
\end{array}$ & Cintia de Lima Garcia & Aceito \\
\hline Outros & AubrizacaoPesquisa.docx & $\begin{array}{c}23 / 05 / 2019 \\
16: 13.25\end{array}$ & Cintia de Lima Garcia & Aceito \\
\hline Outros & CartadeApresentacao docx & $\begin{array}{c}23 / 05 / 2019 \\
16: 13.00\end{array}$ & Cintia de Lima Garcia & Aceito \\
\hline Outros & CartadeAnuencia,docx & $\begin{array}{c}23 / 05 / 2019 \\
16: 11: 17 \\
\end{array}$ & Cintia de Lima Garcia & Aceito \\
\hline $\begin{array}{l}\text { TCLE / Termos de } \\
\text { Assentimento / } \\
\text { Justificativa de }\end{array}$ & TCLE,docx & $\begin{array}{c}23 / 05 / 2019 \\
16: 10: 23\end{array}$ & Cintia de Lima Garcia & Aceito \\
\hline
\end{tabular}

Endereço: Rua Socarro Games, 190

Bairro: Guaien

UF: CE Municipio: FORTALEZA

Telefone: $\langle 85$ ) 3195-2767 Fax: (85)3195-2705 E-mail: cepisghegramailicom 


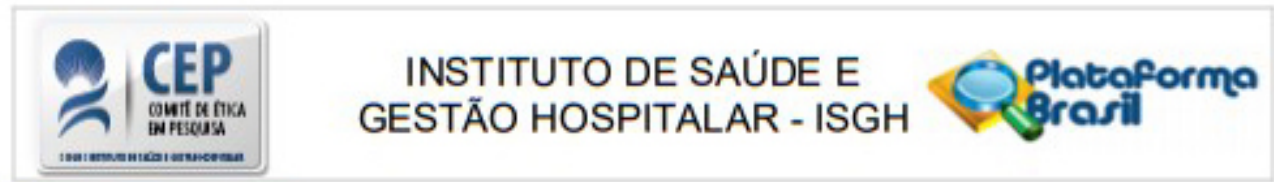

Contanuacto do Parecer. 3.488 .076

\begin{tabular}{|l|l|c|l|l|}
\hline Ausência & TCLE.docx & $\begin{array}{c}23 / 05 / 2019 \\
16: 10: 23\end{array}$ & Cintia de Lima Garcia & Aceito \\
\hline
\end{tabular}

\section{Situaçăo do Parecer:}

Aprovado

Necessita A preciaçăo da CONEP:

Não

FORTALEZA, 06 de Agosto de 2019

\section{Assinado por:}

Jamille Soares Moreira Alves

(Coordenador(a)) 